\title{
Comparative Transcriptome Analysis of the Pinewood Nematode Bursaphelenchus xylophilus Reveals the Molecular Mechanism Underlying Its Defense Response to Host-Derived $\alpha$-pinene
}

\author{
Yongxia Li ${ }^{1,2, \dagger}$, Fanli Meng ${ }^{1,2, \dagger}$, Xun Deng ${ }^{1}$, Xuan Wang ${ }^{1,2}$, Yuqian Feng ${ }^{1,2}$, Wei Zhang ${ }^{1,2}$, \\ Long Pan ${ }^{1,2}$ and Xingyao Zhang ${ }^{1,2, *}$ \\ 1 Laboratory of Forest Pathogen Integrated Biology, Research Institute of Forestry New Technology, \\ Chinese Academy of Forestry, Beijing 100091, China; lyx020419@caf.ac.cn (Y.L.); mfl@caf.ac.cn (F.M.); \\ XunDeng@caf.ac.cn (X.D.); xwang@caf.ac.cn (X.W.); fengyq@caf.ac.cn (Y.F.); zhangwei1@caf.ac.cn (W.Z.); \\ longpan@caf.ac.cn (L.P.) \\ 2 Co-Innovation Center for Sustainable Forestry in Southern China, Nanjing Forestry University, \\ Nanjing 210037, China \\ * Correspondence: xyzhang@caf.ac.cn; Tel.: +86-10-6288-8570 \\ + These authors contributed equally to this work.
}

Received: 17 January 2019; Accepted: 16 February 2019; Published: 20 February 2019

\begin{abstract}
Bursaphelenchus xylophilus is fatal to the pine trees around the world. The production of the pine tree secondary metabolite gradually increases in response to a B. xylophilus infestation, via a stress reaction mechanism(s). $\alpha$-pinene is needed to combat the early stages of $B$. xylophilus infection and colonization, and to counter its pathogenesis. Therefore, research is needed to characterize the underlying molecular response(s) of $B$. xylophilus to resist $\alpha$-pinene. We examined the effects of different concentrations of $\alpha$-pinene on the mortality and reproduction rate of $B$. xylophilus in vitro. The molecular response by which $B$. xylophilus resists $\alpha$-pinene was examined via comparative transcriptomics of the nematode. Notably, B. xylophilus genes involved in detoxification, transport, and receptor activities were differentially expressed in response to two different concentrations of $\alpha$-pinene compared with control. Our results contribute to our understanding of the molecular mechanisms by which B. xylophilus responds to monoterpenes in general, and the pathogenesis of B. xylophilus.
\end{abstract}

Keywords: Bursaphelenchus xylophilus; $\alpha$-pinene; physical and molecular responses; interaction

\section{Introduction}

Pine wilt disease (PWD) is caused by the pine wood nematode (PWN), Bursaphelenchus xylophilus, [1] and is an extremely destructive disease. PWD has caused major losses of forests in European [2-5] and Asian [6-8] countries, especially in Japan, China, South Korea, Portugal, and Spain. It was first identified in Nanjing, Jiangsu Province, China in 1982 [9]. Over the past 30 years, PWD has expanded to 16 provinces and areas in China. In the winter of 2017, PWD spread to Fushun, Liaoning Province, where the annual average temperature is $5-7^{\circ} \mathrm{C}$. Pinus spp. are the main hosts for B. xylophilus [10-12]. Monochamus spp. beetles $[13,14]$ are the major vectors for B. xylophilus infection, as they feed on more than one pine tree during the course of their lives and, thereby, introduce the nematodes into multiple trees through the open sores caused by feeding. Once pine trees are infected, they usually die rapidly, as no effective treatment is available to counteract the disease. Despite many studies concerning PWD and PWNs, the pathogenic mechanism of B. xylophilus has not been completely elucidated. 
As for the pathogenic mechanism(s) of PWD, several hypotheses have been put forward, such as cavitation caused by terpenoid-induced blockage of water transport in xylem tracheids $[15,16]$, phytotoxins produced by PWN-associated bacteria, and cellulose and pectase secreted by PWNs that degrade the cell wall. Cavitation is a typical pathological characteristic of PWD, especially as the disease progresses. Cavities in infected pines may have different physical manifestations, including pine holes [17-19]. One hypothesis that explains how cavitation is involved in the death of PWN-infested trees is that the tracheid cavitation induced by volatile terpenes leads to a water deficit.

Terpenoids are a major type of metabolite of conifers, with monoterpenes being the most important defensive volatile substances, which provide resistance to external plant herbivores and associated pathogen invasion $[20,21]$. $\alpha$-pinene accounts for a large proportion of monoterpenes produced by conifers [22]. Zhao and colleagues [23] found significant differences in the concentrations of $\alpha$-pinene, $\beta$-pinene, and $\beta$-phellandrene in resistant, partially resistant, and sensitive Pinus massoniana, respectively, after inoculation with PWNs, and that the concentrations of the three compounds correlated negatively with $P$. massoniana resistance. $\alpha$-pinene and $\beta$-pinene are the main forms of pinene in pine trees, and are found at a ratio of 1:0.1 [24-26]. Kuroda and colleagues [15] inoculated Pinus thunbergii seedlings with PWNs and analyzed the xylem derivatives produced by the infections. They found that monoterpenes and sesquiterpenes concentrations increased 2 to 4 times in the xylem soon after PWN invasion, and that these compounds permeated the tracheid via the parenchyma cell wall hole, which eventually led to tube cavitation.

A draft genome sequence of B. xylophilus was reported in 2011 [27]. Its availability, in conjunction with previous studies on B. xylophilus, including a large-scale proteome study [28], should facilitate additional studies on PWD pathogenicity. To date, however, only a few transcriptome-type studies on the resistance mechanism(s) of masson pines against PWN have been reported [29,30]. Xu and colleagues [31] demonstrated transcriptional responses to PWN infection in non-resistant masson pines. However, no transcriptome data for the response(s) by B. xylophilus to terpenoid induction has been reported. Therefore, for the study reported herein, we examined the effects of $\alpha$-pinene on the physical and molecular responses of B. xylophilus, to understand the pathogenesis mechanism(s) of this nematode more clearly. We constructed nine cDNA libraries, one that characterized the response(s) of altered transcription of $B$. xylophilus genes at a relative low concentration of $\alpha$-pinene $(\mathrm{AD}, 56.33 \mathrm{mg} / \mathrm{mL}$ ), a second that examined the changes in B. xylophilus gene expression in response to a greater concentration of $\alpha$-pinene (AG, $214.5 \mathrm{mg} / \mathrm{mL}$ ), and a control transcriptome (no $\alpha$-pinene added) for comparison [32]. Genes with altered expression caused by $\alpha$-pinene were identified by comparing transcriptome data, and some of the results for the two $\alpha$-pinene treatments were further studied with real-time quantitative reverse-transcription PCR (qRT-PCR).

\section{Results}

\subsection{Toxic Effects by $\alpha$-Pinene on B. xylophilus Viability and Reproduction}

The toxicity of $\alpha$-pinene on B. xylophilus was determined by the cotton ball assay, with the results showing that the $B$. xylophilus mortality rate was significantly different compared with the control $(p<0.05)$, and that mortality increased with increasing concentrations of $\alpha$-pinene. The highest mortality rate was $22.5 \%$ after the PWNs were treated for $48 \mathrm{~h}$ (Figure 1). In addition, at $42.9 \mathrm{mg} / \mathrm{mL}$, $\alpha$-pinene reduced the PWN reproduction rate, whereas the rate increased at greater concentration $(128.7 \mathrm{mg} / \mathrm{mL}$; Figure 2). The lowest reproduction rate was observed with $56.33 \mathrm{mg} / \mathrm{mL} \alpha$-pinene (Figure 3). 


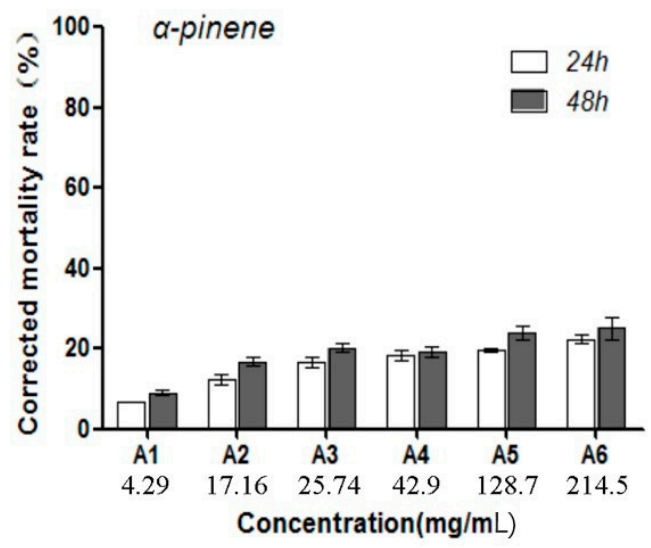

Figure 1. The mortality rates of the pine wood nematodes (PWNs) caused by different $\alpha$-pinene concentrations. For each treatment, the mortality of PWNs exposed to $\alpha$-pinene was corrected for the mortality of counterparts exposed to control using aqueous $0.5 \%$ Triton X-100 $(\mathrm{m} / \mathrm{m})$ solution. There were three biological replications for each experiment. The error line means standard deviation of the mean.
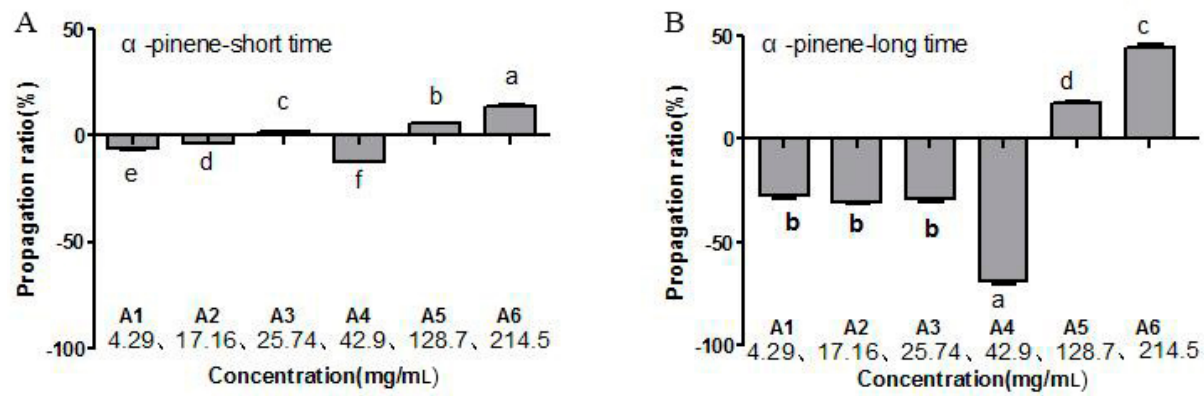

Figure 2. Effect of $\alpha$-pinene concentration and treatment time on the reproduction rate of PWNs. (A) Treatment for a short time. (B) Treatment for a long time. There were three biological replications for each experiment. The error line means standard deviation of the mean. Bars with different letters indicate significant differences among the treatments, as defined by Duncan's test $(p<0.05)$.

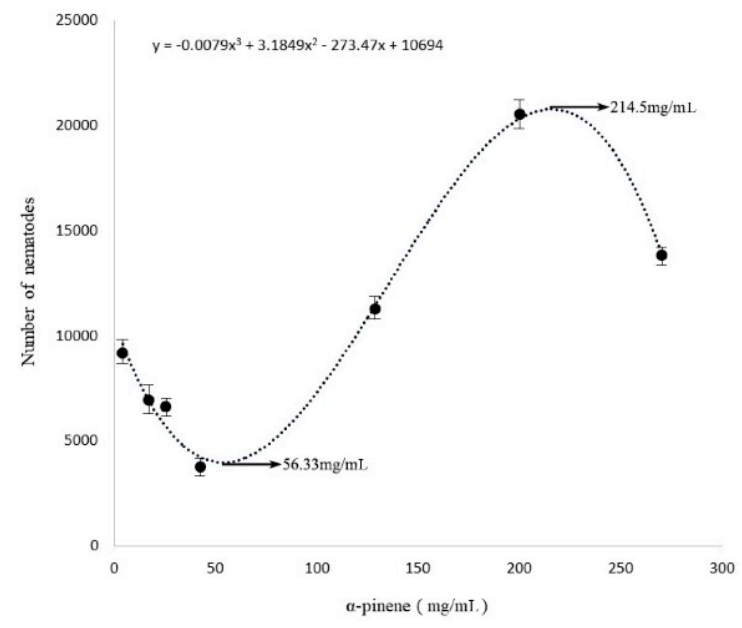

Figure 3. PWN population quantity as affected by $\alpha$-pinene concentration. The points represent the number of nematodes. The line represents the best fit of the data. There were three biological replications for each experiment. The error line means standard deviation of the mean. 


\subsection{Transcriptome Sequencing and Data Assembly}

The total raw reads were generated from the nematode AD (2,798,113,800 bp), AG (2,267,290,600 bp), and CK $(2,121,994,600 \mathrm{bp})$ transcriptomes (Table 1). The raw sequencing data for the CK, AD, and AG samples were deposited into the NCBI sequence read archive database under the B. xylophilus genome sequence (access numbers CADV01000001 to CADV01010432). After removing the adaptor sequences, ambiguous nucleotides, and the low-quality sequences, clean base reads were obtained for the $C K$, AD, and AG samples, with $>85 \%$ of the reads in all nine samples exceeding Q20 and Q30 (Table 1), indicating high-quality sequencing. Meanwhile, the number of unique mapped genes was used to evaluating the gene expression. The number of genes that were effectively expressed were 14,796 (CK), 15,159 (AD) and 14,998 (AG), respectively.

Table 1. Quality of sequencing data for the $\mathrm{AD}, \mathrm{AG}$, and CK samples. Note: $\mathrm{AD}, 56.33 \mathrm{mg} / \mathrm{mL}$ of $\alpha$-pinene. AG, $214.5 \mathrm{mg} / \mathrm{mL}$ of $\alpha$-pinene. CK, control. ExpGene, Expression genes.

\begin{tabular}{ccccccc}
\hline Sample & Data (bp) & Reads & Q20 (\%) & Q30 (\%) & Mapped (\%) & ExpGene \\
\hline CK & $2,121,994,600$ & $21,219,946$ & 96.495 & 89.795 & 96.77 & 14,796 \\
AD & $2,798,113,800$ & $27,981,138$ & 96.705 & 90.255 & 97.06 & 15,159 \\
AG & $2,267,290,600$ & $22,672,906$ & 96.650 & 90.095 & 96.94 & 14,998 \\
\hline
\end{tabular}

\subsection{Differentially Expressed Genes (DEGs) Found Among the Transcriptomes}

A comparison of transcriptome profiles among $\mathrm{CK}, \mathrm{AD}$, and $\mathrm{AG}$, revealed the DEGs of transcriptomes with each other. The results analysis showed that 121 DEGs were specific to AD and 97 DEGs were specific to AG (Figure 4). For the Gene Ontology (GO) "molecular function" category, 10 upregulated DEGs (CK-AD and CK-AG) were significantly enriched, among which five subcategories were potentially involved in environmental adaption, i.e., oxidoreductase activity, membrane ion channel and transport, steroid hormone receptor activity, hedgehog receptor activity, and acid phosphatase activity (Table 2). In addition, 10 DEGs (CK-AD and CK-AG) were notably downregulated, including those related to ATP binding, cytoskeleton, transmembrane transport, and alcohol dehydrogenase activity (Table 2).

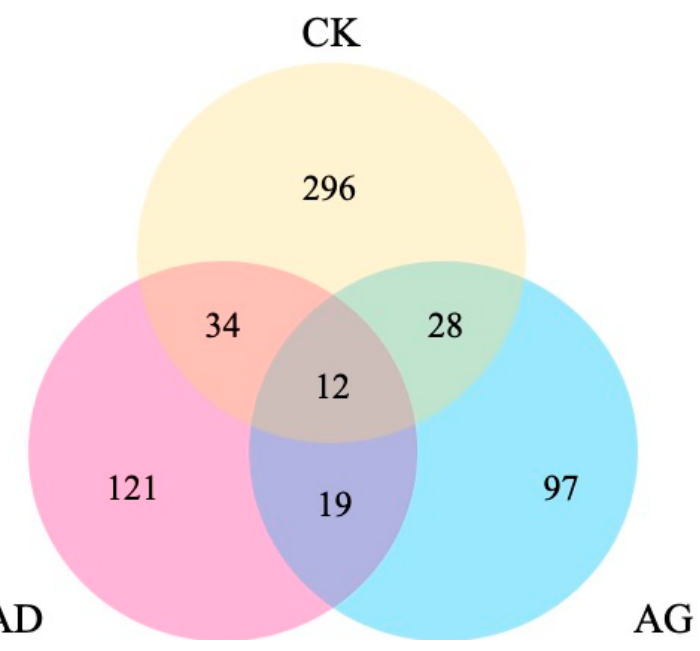

Figure 4. Venn diagram showing the distribution of differentially expressed genes (DEGs) among the transcriptomes. Note: AD, $56.33 \mathrm{mg} / \mathrm{mL}$ of $\alpha$-pinene. AG, $214.5 \mathrm{mg} / \mathrm{mL}$ of $\alpha$-pinene. $\mathrm{CK}$, control. 
Table 2. Upregulated and downregulated Bursaphelenchus xylophilus genes induced by $\alpha$-pinene.

\begin{tabular}{|c|c|c|c|c|}
\hline Condition & ID & Fold Change & $p$-Value & Annotation \\
\hline \multirow{7}{*}{ CK-AD-up } & BUX.s01144.121 & 29.0980 & $2.4758 \times 10^{-13}$ & CYP-33C4,Oxidoreductase \\
\hline & BUX.s00351.319 & 23.1279 & 0.0364 & unc-8,Membrane Ion channel and transport \\
\hline & BUX.s00460.317 & 17.2096 & $3.57851 \times 10^{-6}$ & ugt- 48 ,Glycosyltransferase \\
\hline & BUX.s01063.115 & 15.4635 & $8.46364 \times 10^{-13}$ & CYP-33C2,Oxidoreductase \\
\hline & BUX.s00116.700 & 14.3489 & $1.16933 \times 10^{-11}$ & CYP-33C9,Oxidoreductase \\
\hline & BUX.s00460.348 & 11.4609 & $2.44898 \times 10^{-8}$ & CBG01395,steroid hormone receptor activity \\
\hline & BUX.s00460.315 & 10.2344 & $6.54149 \times 10^{-10}$ & ugt-48,Glycosyltransferase \\
\hline \multirow{6}{*}{ CK-AD-down } & BUX.s01337.103 & 34.2444 & 0.016867135 & Dimethylaniline monooxygenase \\
\hline & BUX.s01147.198 & 33.0521 & $3.56339 \times 10^{-16}$ & Alcohol dehydrogenase 1 \\
\hline & BUX.s00055.304 & 32.8176 & 0.020311806 & CBR-CUTI-1,Unknown \\
\hline & BUX.s01147.18 & 18.8661 & $8.05309 \times 10^{-6}$ & SCAF14537,small GTPase \\
\hline & BUX.s00609.89 & 17.8356 & 0.026861087 & Chia-prov protein (Fragment) \\
\hline & BUX.s01254.326 & 17.5406 & $2.43072 \times 10^{-12}$ & Elo-6,transferase activity \\
\hline \multirow{7}{*}{ CK-AG-up } & BUX.s00116.700 & 13.2087 & $3.07567 \times 10^{-20}$ & CYP-33C9,Oxidoreductase \\
\hline & BUX.s01063.115 & 10.4479 & $3.01485 \times 10^{-19}$ & CYP-33C2,Oxidoreductase \\
\hline & BUX.s01092.190 & 8.7500 & 0.0431 & CBN-SRV-15,Unknown \\
\hline & BUX.s01337.92 & 8.4583 & 0.0493 & PTR-12,hedgehog receptor activity \\
\hline & BUX.s01144.121 & 8.2639 & $3.21387 \times 10^{-7}$ & CYP-33C4,Oxidoreductase \\
\hline & BUX.s00460.348 & 7.5662 & $7.77552 \times 10^{-9}$ & CBG01395,steroid hormone receptor activity \\
\hline & BUX.s00460.317 & 7.3889 & 0.0021 & ugt-48,Glycosyltransferase \\
\hline \multirow{6}{*}{ CK-AG-down } & BUX.s01281.195 & 5.6961 & $1.53 \times 10^{-9}$ & Alcohol dehydrogenase 1 \\
\hline & BUX.s01281.74 & 5.4611 & $8.24 \times 10^{-12}$ & F44E5.4,ATP binding \\
\hline & BUX.s00560.3 & 5.4286 & 0.0034 & TTR-28,extracellular space \\
\hline & BUX.s00364.138 & 5.3184 & $1.22 \times 10^{-10}$ & F27D9.2,transmembrane transport \\
\hline & BUX.s00961.158 & 4.9979 & $7.15 \times 10^{-11}$ & HSP-70,ATP binding \\
\hline & BUX.s00364.193 & 4.7950 & $2.06 \times 10^{-10}$ & Acyl-coenzyme A oxidase; \\
\hline
\end{tabular}

\subsection{Functional Annotation of the DEGs}

Among the upregulated and downregulated genes identified in the transcriptomes, we looked for the major functional PWN genes that might respond to stress induced by $\alpha$-pinene. These genes included those involved in detoxification, transport, and receptor activities.

\subsubsection{DEGs Involved in Detoxification}

Compared with the CK transcriptome, four types of genes encoding the proteins CYP450, UGT, SDR, and GST, which are involved in detoxification, were upregulated or downregulated. A total of 20 and 14 CYP450 genes were found to be differentially regulated in the AD and AG samples, respectively, 14 and 15 UGT genes were found to be differentially regulated in the AD and AG samples, respectively, 14 and 17 SDR genes were found to be differentially regulated in the AD and AG samples, respectively, and three and two GST were found to be differentially regulated in the AD and AG samples, respectively. Cytochrome P450s are heme proteins that play central roles in oxidative metabolism and detoxification. Four unigenes (cyp-33c2, cyp-33c4, cyp-33c9, and cyp-32a1) that best matched CYP450 genes were upregulated in the AD and AG samples (Table 3). 
Table 3. Summary of DEGs encoding detoxification, transport, and receptor proteins.

\begin{tabular}{|c|c|c|c|c|}
\hline \multirow{2}{*}{ Classification } & \multirow{2}{*}{ Genes } & \multirow{2}{*}{ Function } & \multicolumn{2}{|c|}{ Fold Change } \\
\hline & & & CK-AD & $\mathrm{CK}-\mathrm{AG}$ \\
\hline \multirow{16}{*}{ Detoxification } & cyp-32a1 & Monooxygenase activity & 9.4349 & 6.8873 \\
\hline & сур-13a8 & Monooxygenase activity & 0.3504 & 0.4425 \\
\hline & cyp-33c1 & Monooxygenase activity & 6.2375 & 7.2917 \\
\hline & сур-33c2 & Monooxygenase activity & 15.4635 & 10.4479 \\
\hline & cyp-33c4 & Monooxygenase activity & 29.098 & 8.2639 \\
\hline & сур-33c9 & Monooxygenase activity & 14.3489 & 13.2087 \\
\hline & cyp-33e2 & Monooxygenase activity & 0.0336 & 0.2401 \\
\hline & ugt-47 & UDP-glucuronosyl Transferase & 4.4247 & 2.6057 \\
\hline & ugt- 48 & UDP-glucuronosyl Transferase & 17.2096 & 7.3889 \\
\hline & ugt- 49 & UDP-glucuronosyl Transferase & 3.2175 & - \\
\hline & sdr-1 & Dehydrogenase/reductase & 5.011 & 3.325 \\
\hline & sdr-4 & Dehydrogenase/reductase & 0.1948 & 0.3949 \\
\hline & dhs-2 & Dehydrogenase/reductase & 7.528 & 3.8671 \\
\hline & dhs-27 & Dehydrogenase/reductase & 4.4334 & 3.1952 \\
\hline & gst-33 & Glutathione S-Transferase & 0.1312 & 0.4045 \\
\hline & gst-39 & Glutathione S-Transferase & 0.1734 & 0.4042 \\
\hline \multirow{14}{*}{ Transport } & unc- 8 & Ion channel and transport & 23.1279 & - \\
\hline & CBG06849 & Integral to membrane & 4.9059 & 5.0114 \\
\hline & CBG15937 & Integral to membrane & 3.399 & 3.5515 \\
\hline & T09B9.2 & Transmembrane transport & 2.6854 & 3.0404 \\
\hline & TSP-11 & Protein; Transmembrane & 3.6887 & - \\
\hline & TWK-8 & Potassium channel activity & 3.9715 & 2.1194 \\
\hline & ATPase & Sodium/potassium transport & 2.3976 & - \\
\hline & $\mathrm{ABC}$ & $\mathrm{ABC}$ transporter; & 4.5513 & 2.3998 \\
\hline & F44E7.7 & Transmembrane transport & - & - \\
\hline & K09E9.1 & Transmembrane transport & - & 2.254 \\
\hline & F27D9.2 & Transmembrane transport & 0.1588 & - \\
\hline & unc-49 & Channel activity & 2.2265 & - \\
\hline & T19D12.9 & Transmembrane transport & 0.3451 & - \\
\hline & Y19D10A.11 & Transmembrane transport & 0.2002 & - \\
\hline \multirow{15}{*}{ Receptor } & CBG01395 & Steroid hormone receptor activity & 11.4609 & 7.5662 \\
\hline & IFTA-2 & Cell growth and regulatory & 2.2312 & - \\
\hline & Exostosin-2 & Multicellular organismal development & 2.9168 & - \\
\hline & PTR-12 & Hedgehog receptor activity & 12.148 & 8.4583 \\
\hline & PTR-13 & Hedgehog receptor activity & - & 2.1063 \\
\hline & SREBP-1c & Sterol regulatory element-binding & 4.4802 & - \\
\hline & START & START domain protein; cell division & - & - \\
\hline & shr-86 & Steroid hormone receptor activity & 6.7994 & 3.1469 \\
\hline & nhr-19 & Nuclear receptor family member & 2.1711 & - \\
\hline & nhr-3 & Nuclear receptor family member & 2.1206 & - \\
\hline & nhr-40 & Nuclear receptor family member & 2.706 & - \\
\hline & nhr-62 & Nuclear receptor family member & 2.4684 & 2.0637 \\
\hline & nhr-70 & Nuclear receptor family member & 3.1917 & 2.5895 \\
\hline & nhr-10 & Nuclear receptor family member & 2.649 & 2.9513 \\
\hline & nhr-31 & Nuclear receptor family member & - & - \\
\hline
\end{tabular}

\subsubsection{DEGs Involved in Transport}

A large number of genes encoding channels and transporters were upregulated or downregulated in the PWNs treated with both concentrations of $\alpha$-pinene, as compared with the control. The number of upregulated and downregulated genes encoding the channels and transporters was greater in the AD sample than in the AG sample. A B. xylophilus gene that is similar in sequence to Caenorhabditis elegans unc-8 was potentially differentially expressed between conditions. In the AG sample, the unc- 8 gene was expressed more than 23.12 times that of the control. The gene unc- 8 is involved in the transport of metabolized toxic substances and ion balance. Conversely, no significant difference was observed for unc-8 in the AD and CK samples. T09B9.2 is a gene from C. elegans, and is a highly 
expressed member of the transporter superfamily and an important secondary transporter that spans the plasma membrane. A similar sequence was found in B. xylophilus, and this similar sequence was upregulated by 2.68-fold and 3.04-fold in the AD and AG samples, respectively. In addition, genes similar in sequence to those of $C$. elegans encoding ATP-binding cassette (ABC) transporters were upregulated 4.55-fold and 2.39-fold in the AD and AG sample compared with the CK sample. Genes sequence similar to those of $C$. elegans encoding other channels and transporters, e.g., TSP-11, TWK-8, T16G1.3, and K09E9.1, were also upregulated. Conversely, other channels and transporters, e.g., F27D9.2, T19D12.9, and Y19D10A.11, were downregulated (Table 3).

\subsubsection{DEGs Encoding Receptors}

A B. xylophilus gene with similar sequence to the C. elegans PTR-12, a receptor involved in hedgehog signaling, was upregulated 12.14-fold and 8.45-fold in the AD and AG transcriptomes, respectively. CBG01395 is a gene from C. elegans, and a receptor belonging to the nuclear hormone receptor family. A similar sequence is found in B. xylophilus, and the expression of this similar sequence was upregulated 11.46-fold and 7.56-fold in the AD and AG transcriptomes compared with the control. Exostosin-2 is a gene from C. elegans, and has a characterized function in that organism in soft tissue architecture. A similar sequence is found in B. xylophilus, and this similar sequence is differentially regulated-how that tenuous link relates to function in B. xylophilus during parasitism is not really clear. The gene encoding exostosin-2 was upregulated 2.91-fold in the AD sample. In addition, the genes that are similar in sequence to those of $C$. elegans encoding the acetylcholine receptor subunit alpha-type deg-3, the cell-growth-regulating nucleolar protein, and the sterol regulatory-element-binding protein 1 were upregulated in the AD sample. Nuclear receptors are primarily responsible for transmitting extracellular signals that regulate the expression of specific genes for control of cell development, stabilization, and metabolism. Genes with similar sequence to those of $C$. elegans encoding nuclear receptors, including shr-86, nhr-19, nhr-3, nhr- 40 , nhr-62, nhr-70, and nhr-10, were upregulated in the AD sample, and shr-86, nhr-70, nhr-62, and nhr-10 were also upregulated in the AG sample (Table 3).

\subsection{GO Enrichment Analysis of the DEGs}

For functional annotation and classification, all DEGs were subjected to GO enrichment analysis. Upregulated genes and downregulated genes were assigned GO IDs and categories (Figure 5). The main GO categories included cellular component, molecular function, and biological process. For the AD sample, GO assignments were as follows. For the biological process category, $18.53 \%$ of the DEGs were assigned to metabolic processes (GO: 0008152), and 8.88\% were assigned to monomelic metabolic processes (GO: 0044710). For the molecular function category, 54.31\% of the DEGs were assigned to catalytic activity (GO: 0003824 ) and $17.88 \%$ were assigned to redox enzyme activity (GO: 0016491). For the cellular component, $15.78 \%$ of the DEGs were assigned among the extracellular domain (GO: 0005576) and the endoplasmic reticulum (GO: 0005783) categories. In the AG sample, DEGs were assigned as follows. For the biological process category, 26.21\% of the DEGs were assigned to metabolic processes (GO: 0008152) and $11.76 \%$ to monomelic metabolic processes (GO: 0044710). For the molecular function category, $56.77 \%$ of the DEGs were assigned to catalytic activity (GO: 0003824), and 20.00\% to redox enzyme activity (GO: 0016491). For the cellular component category, $13.33 \%$ of the DEGs were assigned to extracellular domain (GO: 0005576) or epidermal protein (GO: 0042302). 
A

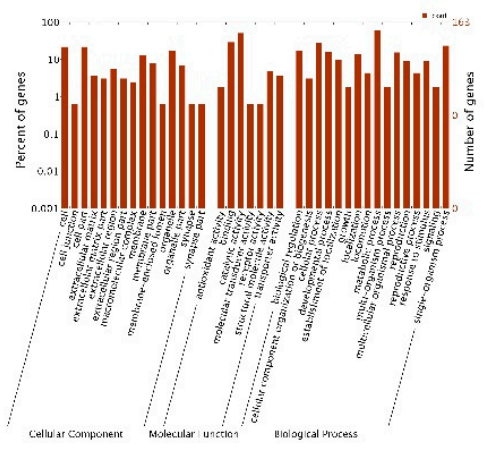

13

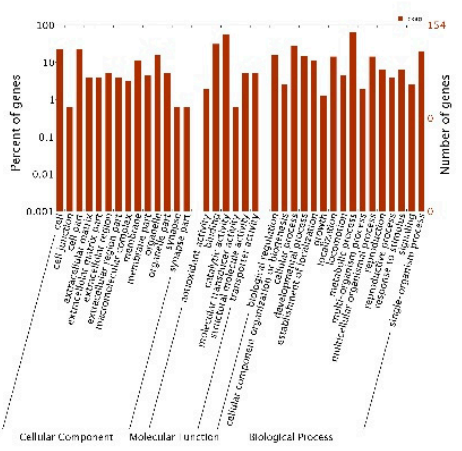

Figure 5. GO assignment of the DEGs in the AD and AG transcriptomes. DEGs in the AD sample (A) and in the AG sample (B) had GO ID assignments mainly in three categories: cellular component, molecular function, and biological process.

\subsection{KEGG Functional Annotation of the DEGs}

KEGG functional annotation for the two treatments revealed that 288 and 249 DEGs were enriched in 34 and 44 KEGG pathways, in the AD and AG samples, respectively (Table 4). The major enriched metabolic pathways included those involved in xenobiotic biodegradation and metabolism, carbohydrate metabolism, lipid metabolism, amino acid metabolism, and metabolism of cofactors and vitamins. In addition, transport and catabolism pathways were important for the category cytology process. The endocrine system, digestive, system, and immune system were also significantly enriched in each treatment.

A total of 114 and 112 DEGs were enriched in the top 25 KEGG pathways in the AD and AG samples, respectively, with half of those DEGs involved in xenobiotic biodegradation and metabolism. Genes for CYP450, UGT, and SDR were upregulated in KEGG pathways for metabolism of xenobiotics by cytochrome P450 (ko00980, see Supplementary Figure S1), aminobenzoate degradation (ko00627, see Supplementary Figure S2), drug metabolism - cytochrome P450 (ko00982, see Supplementary Figure S3), and drug metabolism—other enzymes (ko00983, see Supplementary Figure S4).

Table 4. Top 13 pathways involving DEGs in the AD and AG samples.

\begin{tabular}{ccc}
\hline KEGG (KO) Term & \multicolumn{2}{c}{ NO. Genes } \\
\cline { 2 - 3 } Metabolism & CK-AD & CK-AG \\
\hline Carbohydrate metabolism & 67 & 90 \\
Energy metabolism & 14 & 18 \\
Lipid metabolism & 77 & 66 \\
Amino acid metabolism & 38 & 68 \\
Glycan biosynthesis and metabolism & 16 & 17 \\
Metabolism of cofactors and vitamins & 42 & 53 \\
Metabolism of terpenoids and polyketides & 3 & 4 \\
Biosynthesis of other secondary metabolites & 11 & 13 \\
Xenobiotics biodegradation and metabolism & 114 & 112 \\
\hline Cellular processes & & \\
Transport and catabolism & 57 & 26 \\
Organismal systems & & \\
Immune system & 8 & \\
Digestive system & 12 & 9 \\
Endocrine system & 14 & \\
\hline
\end{tabular}




\subsection{Verification of Gene Expression by $q R T-P C R$}

To confirm the validity of the transcriptome data, we selected a few potentially key DEGs identified in the $\mathrm{AD}$ and $\mathrm{AG}$ samples that encode detoxification, transport, and receptor proteins for analysis with qRT-PCR. The expression patterns from qRT-PCR were compared with the results of the RNA sequencing expression analysis. The results showed that all the 12 genes had the same expression patterns in the qRT-PCR analysis as in the RNA sequencing analysis, which proved the reliability and accuracy of the RNA sequencing expression analysis (Figure 6).
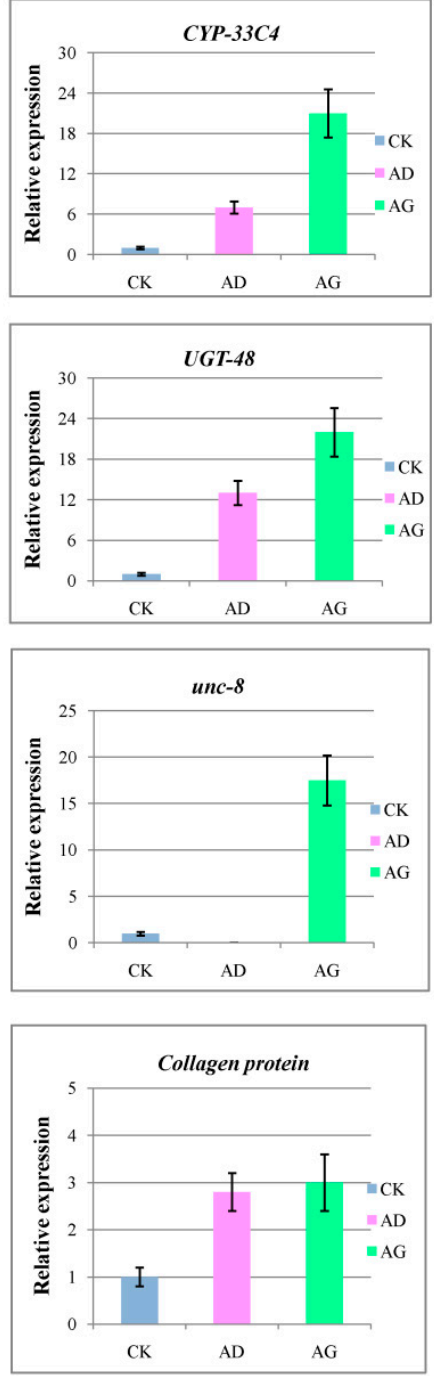
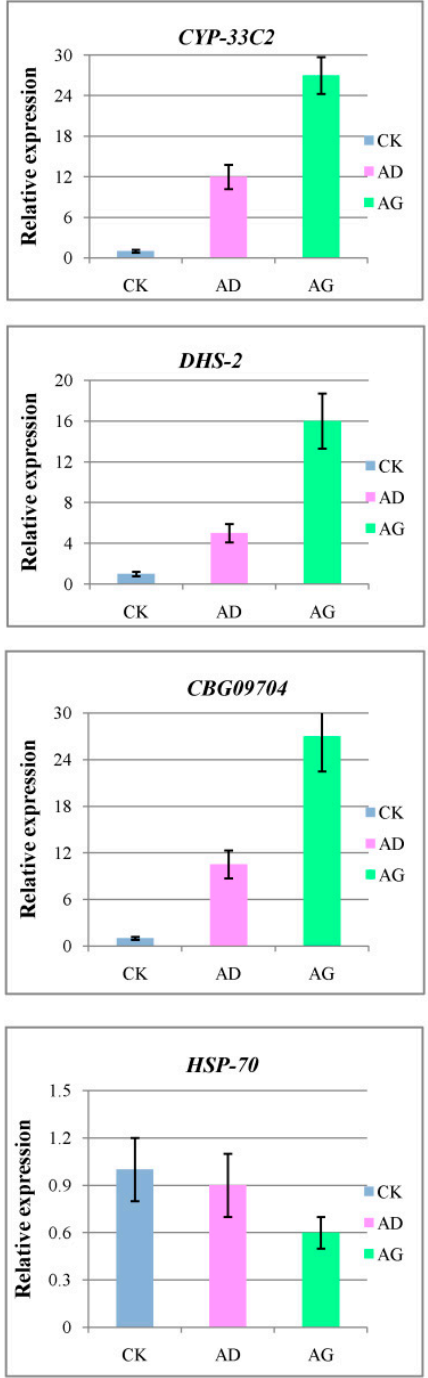
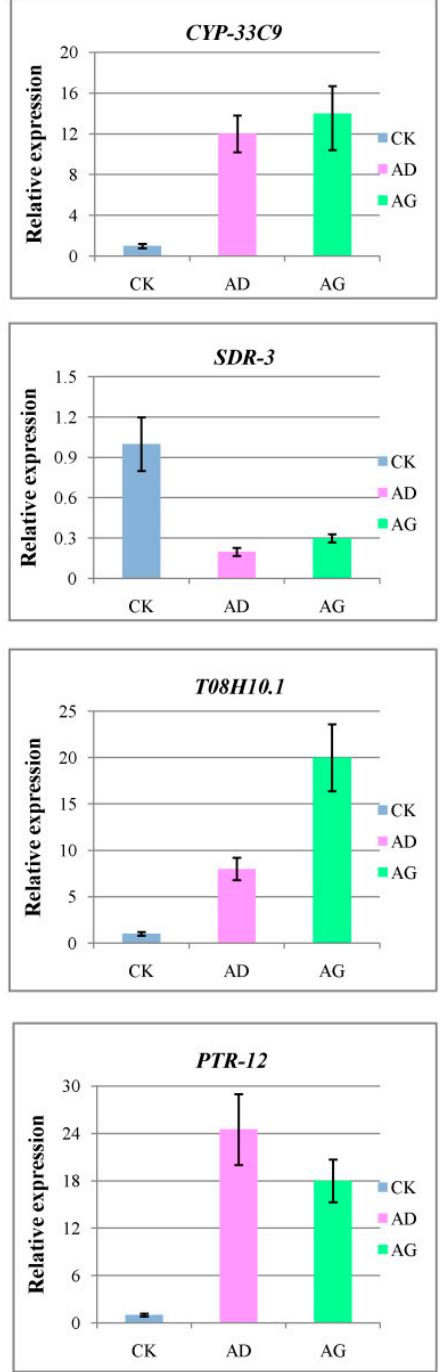

Figure 6. qRT-PCR validation of key candidate DEGs in the AD and AG samples. The CK sample served as the control. Data from the qRT-PCR represent the mean of three replicates, and bars represent standard error.

\section{Discussion}

Terpenes are volatile metabolites that enhance the resistance of plants to biotic stresses, e.g., fungi, bacteria, insects, and nematodes. In this study, the effects of different concentrations of the monoterpene, $\alpha$-pinene, on the mortality and reproduction rates of the PWN were first assessed to determine the optimal $\alpha$-pinene concentration that would kill PWNs in vitro. The results of our biological assay indicated that the reproductive rate of $B$. xylophilus was inhibited at a low concentration of $\alpha$-pinene, but enhanced at a greater concentration. Kong and colleagues [33] identified the main bioactive component of red thyme oil as $\alpha$-pinene and assessed its effects on the mortality and 
reproduction rates of PWNs, revealing that the PWN reproduction rate was inhibited, with an $\mathrm{LC}_{50}$ for $\alpha$ - and $\beta$-pinene of $>20 \mathrm{mg} / \mathrm{mL}$. Niu [26] assessed the effects of different concentrations of $\alpha$-and $\beta$-pinene on the PWN and found that each compound inhibited PWN viability at a small concentration and promoted reproduction at a large concentration. The aforementioned results reveal that terpenoids can impact the health of PWNs. Our analysis showed that there was a trough and a peak in the fit of the line to the PWN population quantity as affected by $\alpha$-pinene concentration (Figure 3 ). Therefore, it was important that we determine the optimal concentration of $\alpha$-pinene with which to treat B. xylophilus in vitro based on the physiological index, $56.33 \mathrm{mg} / \mathrm{mL}(\mathrm{AD})$ and $214.5 \mathrm{mg} / \mathrm{mL}(\mathrm{AG})$ of $\alpha$-pinene, prior to performing our transcriptome experiments.

We constructed two transcriptome databases from total B. xylophilus RNA after exposing the nematodes to $\alpha$-pinene. The expression of certain genes in the $\alpha$-pinene-treated samples was upregulated, including those encoding oxidoreductase, membrane ion channels and transport proteins, steroid hormone receptors, hedgehog receptors, acid phosphatases, glycosidases, and hydrolases. The expression of certain other genes was downregulated, including those encoding ATP-binding proteins, cytoskeletal proteins, transmembrane transport proteins, alcohol dehydrogenases, histone acetyltransferases, acyl-CoAs, oxidases, $\alpha$-(1,3)-fucosyltransferase $\mathrm{c}$, and transferases.

The expression of certain genes was upregulated after the nematodes were exposed to $\alpha$-pinene, including those of the CYP450, UGT, and SDR families. The PWN is a plant parasite, and its metabolite that is specifically related to detoxification is essential if it is to resist host systemic and induced responses [34]. The PWN completes its parasitic process primarily in the resin of the pine tree. Metabolites found in the pine tree that are toxic to PWNs include terpenoids [35] and aromatic compounds [36]. Genome sequencing analysis of B. xylophilus [27] revealed that certain detoxification proteins can reduce the harm caused by host secondary products, and ensure normal feeding and migration so that they can complete their entire life cycle. As previously reported, CYP450s are enriched in the nematode and mediate the degradation of xenobiotics $[27,37]$. Notably, pinenes are the most abundant monoterpenoids in pine trees. A crude sulfate turpentine from the pine tree was found to be composed of $60-65 \% \alpha$-pinene and 25-35\% $\beta$-pinene [38]. According to the research of Espada, CYP450 and UGTs were up-regulated at the early stages of infection, and were involved in the detoxification of xenobiotic compounds ( $\alpha$-pinene and $\beta$-pinene) [39]. Therefore, to thrive on pine trees, PWNs must have effective mechanisms to degrade pinenes. CYP450 and SDR participate in the phase I detoxification process, and UGT and GST participate in the phase II detoxification process. We found that UGT genes were upregulated and GST and SDR genes were downregulated in the AD and AG samples, suggesting that the CYP450 and UGT activities help PWNs cope with $\alpha$-pinene stress by detoxifying it. We also used qRT-PCR to amplify three CYP450 genes (CYP-33C9, CYP-33C4, CYP-33C2; Figure 5), the encoded proteins of which are secreted and have an oxidoreductase or transferase activity. Our results will be important for researchers who study detoxification proteins.

Cheng and colleagues [40] showed that PWNs may not be able to completely degrade terpenes, which can only be excreted through redox modification. They also found that bacteria associated with PWNs are needed for complete degradation of terpenes. Detoxification proteins were found to be involved in redox metabolism of terpenes; moreover, channels and transporters appear to be necessary to expel terpenes. For survival, the nematode body must reconcile the difference in external osmotic pressure and respond productively to environmental stress. Unc-8 is a channel protein that maintains the balance between internal and external cellular osmotic pressure and acts as an ion channel in Caenorhabditis elegans [41]. Expression of the unc-8 gene was significantly upregulated in the AG sample, suggesting its participation in detoxification processes. In addition, the genes encoding CBG09704 [42] and T08H10.1 [43] were also upregulated in the AG samples. Given our results, certain PWN genes were upregulated and genes for certain channels were downregulated to ensure efficient detoxification and transport to cope with terpene stress.

In addition to DEGs related to detoxification and transport for which expression was altered, the expression of certain genes encoding receptors was upregulated by $\alpha$-pinene treatment; the protein 
products of these genes are involved in normal growth processes or are metabolism-related hormones or stress response proteins. Apparently, in our study, these genes were differentially expressed in PWNs, so that healthy conditions could be maintained during the adverse physiological stress induced by $\alpha$-pinene. The Hedgehog signaling pathway plays a key role in embryonic development, as it helps regulate cell proliferation and differentiation and coordinates tissue and organ development. This signaling pathway is evolutionarily conserved, as it is needed to regulate vertebrate and invertebrate development [44-46]. PTR-12 is a hedgehog receptor with growth regulatory functions and regulates the molting cycle of $C$. elegans, epidermal protein activity, and the growth of multicellular tissues [47]. These functions suggest that this growth regulatory protein in PWNs may respond to the presence of $\alpha$-pinene. Meanwhile, we selected these potentially key DEGs identified in the AD and AG samples that encode detoxification, transport, and receptor proteins for analysis with qRT-PCR. The expression profiles of the qRT-PCR-amplified genes were very similar to the GO and KEGG functional annotations of the DEGs. According to the GO and KEGG terms found for the DEGs in our study, B. xylophilus responds to $\alpha$-pinene stress by redox-regulated detoxification, with the products being exported via ion or small molecule transport proteins. In addition, metabolic pathways play important roles in the response by PWNs to $\alpha$-pinene stress. Finally, other genes were upregulated in response to $\alpha$-pinene, including those for cuticle synthesis and encoding steroid hormone receptors and hedgehog receptors to ensure normal physiological activities of B. xylophilus.

Our study should provide a foundation for further verification of gene functions, artificial inoculation, RNA interference, and recombinant expression studies in vitro relevant to the defense mechanisms of PWN.

\section{Materials and Methods}

\subsection{Materials}

The highly virulent B. xylophilus strain NXY61 was isolated from wood chips of infested P. massoniana in Zhejiang, China and stored at the Laboratory of Forest Pathogen Integrated Biology of Chinese Academy of Forestry, Beijing, China. The Botrytis cinerea strain has been maintained in our laboratory.

\subsection{Toxic Effects of Different $\alpha$-Pinene Concentrations on the Mortality and Reproduction Rates of B. xylophilus}

Treatment was carried out for a short time via the soak method. Six concentrations of $\alpha$-pinene $(4.29,17.16,25.74,42.9,128.7$, and $214.5 \mathrm{mg} / \mathrm{mL})$ [48] were prepared by serial dilution with distilled water containing $0.5 \%(w / w)$ Triton X-100. One concentration of each $\alpha$-pinene solution and $~ 500$ PWNs (a mixture of juveniles and adults) in $200 \mu \mathrm{L}$ of water were individually introduced into wells of 96 well plates (Falcon, New York, NY, USA). Control samples contained $0.5 \%(w / w)$ Triton X-100 in distilled water and PWNs. The nematodes were maintained under the same environmental conditions $\left(25^{\circ} \mathrm{C}\right.$ for $24 \mathrm{~h}$ and $48 \mathrm{~h}$ in the dark) as used for colony maintenance [33]. The number of nematodes remaining alive was examined under a microscope $24 \mathrm{~h}$ and $48 \mathrm{~h}$ after the start of the experiment. Nematodes were considered to have died when their bodies were straight and did not move even after mechanical prodding.

Treatment for long time carried out the cotton ball bioassay [33], which was used to determine whether $\alpha$-pinene inhibited or stimulated the propagation of B. xylophilus. Briefly, suspensions of nematodes in water ( 6000 nematodes $/ \mathrm{mL})$ were prepared by diluting the standard nematode suspension. Solutions of $\alpha$-pinene (50 $\mu \mathrm{L}$ each) at different concentrations $(4.29,17.16,25.74,42.9,128.7$, and $214.5 \mathrm{mg} / \mathrm{mL}$ ) were individually injected into $5 \mathrm{~mm}$ diameter cotton balls that were separately placed at the center of a mat in $9 \mathrm{~cm}$ diameter Petri dishes, and a nematode suspension $(60 \mu \mathrm{L})$ was then injected into each ball. Controls received an aqueous $0.5 \%$ Triton X-100 $(\mathrm{m} / \mathrm{m})$ solution and a nematode 
suspension. Treated and control nematodes were kept at $25^{\circ} \mathrm{C}$ for $7 \mathrm{~d}$ in the dark. The nematodes were separated from the culture by the Baermann funnel technique and were counted.

\subsection{Preparation of PWNs Using Different Concentrations of $\alpha$-Pinene for Transcriptome Analyses}

Given the results discussed in the first section, the $56.33 \mathrm{mg} / \mathrm{mL}(\mathrm{AD})$ and $214.5 \mathrm{mg} / \mathrm{mL}$ (AG) concentrations of $\alpha$-pinene in $0.5 \%(w / w)$ Triton X-100 were chosen as representative of a small and large concentration, respectively. Controls received an aqueous $0.5 \%$ Triton X-100 $(\mathrm{m} / \mathrm{m})$ solution. PWNs ( 3000 nematodes $/ \mathrm{mL})$ and one of the two representative concentrations or the control solution was subjected to gentle shaking at $25^{\circ} \mathrm{C}$ for $48 \mathrm{~h}$. The samples were then centrifuged for $5 \mathrm{~min}$ at $5000 \times g$ to collect the nematodes for RNA isolation and cDNA synthesis as described in the next section. Each sample was replicated three times.

\subsection{Total RNA Isolation and cDNA Synthesis}

Total RNA from B. xylophilus that had been treated with or without $\alpha$-pinene as described in previous section was extracted with RNAprep Pure Tissue kit reagents (Tiangen Biotech, Beijing, China) according to the manufacturer's protocol. RNA integrity was confirmed using an Agilent 2100 Bioanalyzer (Mainz, Germany) with a minimum RNA integrity number of 8 . The transcriptome samples were prepared using reagents from an Illumina kit (San Diego, CA, USA) following the manufacturer's instructions. Briefly, poly(A) mRNA was purified from total RNA using oligo (dT) magnetic beads, and then fragmented into short sequences in fragmentation buffer. The cleaved poly(A) RNA fragments were used for first-strand cDNA synthesis with random hexamer primers. The second-strand cDNA synthesis used RNase $\mathrm{H}$ and DNA polymerase I. After end repair and adaptor ligation, the products were purified and enriched via PCR to create cDNA libraries.

\subsection{Sequencing and Assemblage of B. xylophilus Transcriptome DEGs in Response to $\alpha$-Pinene}

Each sample was replicated three times (nine cDNA libraries), and the data of raw reads was averaged over three replications. The nine cDNA libraries were sequenced at the Beijing Genome Institute (Shenzhen, China) on an Illumina HiSeq 2000 platform. Raw Illumina reads were quality-trimmed and filter to remove low quality reads using Trimmomatic v. 0.33 (RWTH Aachen University, Aachen, Germany). Filtered reads were aligned to the reference genome using the transcriptome aligner Tophat v. 2.0.13 (Broad Institute of MIT and Harvard, Cambridge, MA, USA). The Htseq-count function from HTSeq v 0.6.1 (Genome Biology Unit, Heidelberg, Germany) was used to calculate the gene counts. Htseq-count would exclude the mapped reads generated form Tophat with more than one reported alignment. Only the unique mapped reads were used in the downstream analysis. Differential expression analysis of all samples was performed using the DESeq R package (1.10.1) based on the negative binomial distribution [49]. The resulting p-values were adjusted using the Benjamini and Hochberg's approach, and the p-adjusted value (padj < 0.05 ) was set as the threshold for significant differential expression [50]. Translated transcript sequences were first searched with Blastx (http:/ / blast.ncbi.nlm.nih.gov/Blast.cgi, accessed on: 20 March 2017) in the non-redundant (nr), Swiss-Prot and KEGG protein databases to retrieve proteins with the greatest sequence similarities, along with their functional annotations. The nr annotations, GO annotations, and functional classifications for the transcripts were characterized using Blast2GO [51,52] and WEGO [53]. Pearson's correlation coefficient was used to evaluate the correlations with respect to the percentages of transcript representations linked to each GO term and KEGG biological pathway for the three transcriptomes.

\subsection{Identification of Statistically Enriched GO Terms and KEGG Pathways}

The hypergeometric test was used to measure significantly enriched GO terms in the target gene groups in comparison with the control $[54,55]$. The formula used to measure significance was 
$p=1-\sum_{i=0}^{m-1} \frac{\left(\begin{array}{c}M \\ i\end{array}\right)\left(\begin{array}{c}N-M \\ n-i\end{array}\right)}{\left(\begin{array}{c}N \\ n\end{array}\right)}$, where $\mathrm{N}$ is the number of all genes with a $\mathrm{GO}$ annotation, $\mathrm{n}$ is the number of DEGs in N, M is the number of genes that are annotated to a certain GO term, and $\mathrm{m}$ is the number of DEGs in M. The GO terms with the $p$-value cut-off of 0.005 were deemed to be enriched. In addition, to identify enriched pathways, the hypergeometric test was used in a similar manner to measure the relative coverage of the annotated KEGG orthologous groups of pathways in the background, and pathways with a $p$-value cut-off of 0.005 were considered enriched [56].

\subsection{Quantitative RT-PCR}

Identical portions of each RNA sample were used for qRT-PCR and transcriptome sequencing. The primer pairs (see Supplementary Table S1) for the candidate genes were designed using Primer Premier 6.0. An ABI 7500 Real-Time PCR instrument (Applied Biosystems, Foster City, CA, USA) in conjunction with the SYBR Green detection method were used for qRT-PCR. The cycling parameters were one round at $95^{\circ} \mathrm{C}$ for $60 \mathrm{~s}$, and then 40 cycles at $95^{\circ} \mathrm{C}$ for $15 \mathrm{~s}$ and $60^{\circ} \mathrm{C}$ for $35 \mathrm{~s}$. The relative expression of each candidate gene was calculated using the $2^{-\Delta \Delta \mathrm{Ct}}$ method [57].

\subsection{Statistical Analysis}

All experiments involving PWNs were independently performed three times, with each treatment replicated five times, and qRT-PCR for each sample was replicated three times. Statistical analyses were performed using SPSS 17.0 (SPSS Inc., Chicago, IL, USA) and Origin 8.0 (OriginLab, Northampton, MA, USA). One-way analysis of variance by Duncan test was used to determine the statistical significance between treatments, with significance being defined as $p<0.05$.

\section{Conclusions}

We examined the effects of different concentrations of $\alpha$-pinene on the mortality and reproduction rate of $B$. xylophilus in vitro. The molecular response by which B. xylophilus resists $\alpha$-pinene was examined via comparative transcriptomics of the nematode. Besides, the genes of $B$. xylophilus involved in detoxification, transport, and receptor activities were differentially expressed in response to two different concentrations of $\alpha$-pinene compared with control. This is beneficial to better understanding the molecular mechanisms of by which B. xylophilus responds to monoterpenes in general, and the pathogenesis of B. xylophilus.

Supplementary Materials: Supplementary materials can be found at http://www.mdpi.com/1422-0067/20/4/ $911 / \mathrm{s} 1$.

Author Contributions: Experimental design: Y.L. and X.Z. Experimental implementation: F.M. and X.D. Material contribution: X.W., Y.F., W.Z. and L.P. Data analysis and manuscript writing: F.M. and Y.L.

Funding: The present research was funded by National Key R\&D Program of China (2017YFD0600102).

Conflicts of Interest: The authors declare there is no competing interest.

\section{Abbreviations}

PWD Pine wilt disease

PWN Pine wood nematode

GO Gene Ontology

KEGG Kyoto Encyclopedia of Genes and Genomes 


\section{References}

1. Nickle, W.R.; Golden, A.M.; Mamiya, Y.; Wergin, W.P. On the taxonomy and morphology of the pine woodnematode, Bursaphelenchus xylophilus (Steiner \&Buhrer 1934) Nickle 1970. J. Nematol. 1981, 13, $385-393$. [PubMed]

2. Branco, M.; Bragança, H.; Sousa, E.; Phillips, A.J. Pests and Diseases in Portuguese Forestry: Current and New Threats; Springer: New York, NY, USA, 2014.

3. Mota, M.; Oliveira, S.; Vicente, C.; Vieira, P. Pine wilt disease in portugal: Recent progress and new approaches to the understanding and control of the pine wood nematode and its insect vector. J. Nematol. 2009, 41, 361 .

4. Abelleira, A.; Picoaga, A.; Mansilla, J.P.; Aguin, O. Detection of Bursaphelenchus xylophilus, causal agent of pine wilt disease on Pinus pinaster in northwestern Spain. Plant Dis. 2011, 95, 776. [CrossRef] [PubMed]

5. Vicente, C.; Espada, M.; Vieira, P.; Mota, M. Pine Wilt Disease: A threat to European forestry. Eur. J. Plant Pathol. 2012, 133, 89-99. [CrossRef]

6. Mamiya, Y. History of pine wilt disease in Japan. J. Nematol. 1988, 20, 219-226. [PubMed]

7. Togashi, K.; Shigesada, N. Spread of the pinewood nematode vectored by the Japanese pine sawyer: Modeling and analytical approaches. Popul. Ecol. 2006, 48, 271-283. [CrossRef]

8. Yoshimura, A.; Kawasaki, K.; Takasu, F.; Togashi, K.; Futai, K.; Shigesada, N. Modeling the spread of wilt disease caused by nematodes with pine sawyers as vector. Ecology 1999, 80, 1691-1702. [CrossRef]

9. Sun, Y.C. Pine wood nematode found at Dr. Sun Yat's Mausoleum in Nanjing. Jiangsu For. Sci. Technol. 1982, 4,47 .

10. Kiyohara, T.; Tokushige, Y. Inoculation experiments of a nematode, Bursaphelenchus sp., onto pine trees. J. Jpn. For. Soc. 2008, 53, 210-218.

11. Mamiya, Y.; Enda, N. Transmission of Bursaphelenchus Lignicolus (Nematoda: Aphelenchoididae) by Monochamus Alternatus (Coleoptera: Cerambycidae). Nematologica 1972, 18, 159-162. [CrossRef]

12. Mota, M.M.; Braasch, H.; Bravo, M.A.; Penas, A.C.; Burgermeister, W.; Kai, M.; Sousa, E. First report of Bursaphelenchus xylophilus in Portugal and in Europe. Nematology 1999, 1, 727-734.

13. Morimoto, K.; Iwasaki, A. Role of Monochamus alternatus (Coleoptera: Cerambycidae) as a vector of Bursaphelenchus lignicolus (Nematoda : Aphelenchoididae). J. Jpn. For. Soc. 1972, 54, 177-183.

14. Ryss, A.; Vieira, P.; Mota, M.; Kulinich, O. A synopsis of the genus Bursaphelenchus Fuchs, 1937 (Aphelenchida: Parasitaphelenchidae) with keys to species. Nematology 2005, 7, 393-458.

15. Kuroda, K.; Yamada, T.; Mineo, K.; Tamura, H. Effects of cavitation on the development of pine wilt disease caused by Bursaphelenchus xylophilus. Jpn. J. Phytopathol. 1988, 54, 606-615. [CrossRef]

16. Kuroda, K. Mechanism of cavitation development in the pine wilt disease. For. Pathol. 2010, $21,82-89$. [CrossRef]

17. Ikeda, T.; Ohtsu, M. Detection of xylem cavitation in field-grown pine trees using the acoustic emission technique. Ecol. Res. 1992, 7, 391-395. [CrossRef]

18. Kuroda, K.; Kanbara, Y.; Inoue, T.; Ogawa, A. Analysis of NMR-CT images to detect the xylem dysfunction and lesions in tree trunks. In Abstracts Pacific Regional Wood Anatomy Conference; FAO the United Nations Regional Office for Asia Pacific (FAO RAP): Bangkok, Thailand, 2002.

19. Utsuzawa, S.; Fukuda, K.; Sakaue, D. Use of magnetic resonance microscopy for the nondestructive observation of xylem cavitation caused by pine wilt disease. Phytopathology 2005, 95, 737-743. [CrossRef] [PubMed]

20. Zulak, K.G.; Bohlmann, J. Terpenoid biosynthesis and specialized vascular cells of conifer defense. J. Intergr. Plant. Biol. 2010, 52, 86-97. [CrossRef]

21. Lewinsohn, E.; Savage, T.J.; Croteau, M.G.R. Simultaneous analysis of monoterpenes and diterpenoids of conifer oleoresin. Phytochem. Anal. 1993, 4, 220-225. [CrossRef]

22. Ning, T.; Fan, J.; Fang, Y.; Sun, J. Changes in contents of host volatile terpenes under different damaged states and electroantennogram response of Monochamus alternatus Hope to these volatiles. Acta Entomol. Sin. 2006, 49, 179-188.

23. Zhen, Z.; Xi, H.U.; Dong, L.I.; Fu, X.U.; Gui, H.U.; Zhen, S.; Xian, L. Study on contents variation of neutral terpenoids of resistant provenance of P. massoniana after inoculating Bursaphelenchus xylophilus. Chem. Ind. For. Prod. 2001, 21, 52-58. 
24. Futai, K. Pine wood nematode, Bursaphelenchus xylophilus. Annu Rev. Phytopathol. 2013, 51, 61-83. [CrossRef] [PubMed]

25. Zhao, L.L.; Wei, W.; Kang, L.; Sun, J.H. Chemotaxis of the pinewood nematode, Bursaphelenchus xylophilus, to volatiles associated with host pine, Pinus massoniana, and its vector Monochamus alternatus. J. Chem. Ecol. 2007, 33, 1207-1216. [CrossRef] [PubMed]

26. Niu, H.; Zhao, L.; Lu, M.; Zhang, S.; Sun, J. The ratio and concentration of two monoterpenes mediate fecundity of the pinewood nematode and growth of Its associated fungi. PLoS ONE 2013, 7, e31716. [CrossRef] [PubMed]

27. Kikuchi, T.; Cotton, J.A.; Dalzell, J.J.; Hasegawa, K.; Kanzaki, N.; McVeigh, P.; Takanashi, T.; Tsai, I.J.; Assefa, S.A.; Cock, P.J.; et al. Genomic insights into the origin of parasitism in the emerging plant pathogen Bursaphelenchus xylophilus. PLoS Pathog. 2011, 7, e1002219. [CrossRef] [PubMed]

28. Ryoji, S.; Hironobu, M.; Taisei, K.; Yuko, T.; Mitsuyoshi, U.; Kazuyoshi, F. Secretome analysis of the pine wood nematode Bursaphelenchus xylophilus reveals the tangled roots of parasitism and Its potential for molecular mimicry. PLoS ONE 2013, 8, e67377.

29. Matsye, P.D.; Kumar, R.; Hosseini, P.; Jones, C.M.; Tremblay, A.; Alkharouf, N.W.; Matthews, B.F.; Klink, V.P. Mapping cell fate decisions that occur during soybean defense responses. Plant Mol. Biol. 2011, 77, 513-528. [CrossRef] [PubMed]

30. Matsye, P.D.; Lawrence, G.W.; Youssef, R.M.; Kim, K.H.; Lawrence, K.S.; Matthews, B.F.; Klink, V.P. The expression of a naturally occurring, truncated allele of an $\alpha$-SNAP gene suppresses plant parasitic nematode infection. Plant Mol. Biol. 2012, 80, 131-155. [CrossRef] [PubMed]

31. Xu, L.; Liu, Z.Y.; Zhang, K.; Lu, Q.; Liang, J.; Zhang, X.Y. Characterization of the Pinus massoniana transcriptional response to Bursaphelenchus xylophilus infection using Suppression Subtractive Hybridization. Int J. Mol. Sci. 2013, 14, 11356-11375. [CrossRef]

32. Cui, J.; Li, Y.; Zhang, W.; Wang, X.; Pan, L.; Feng, Y.; Zhang, X. The population strucuture and sex ratios of Bursaphelenchus xylophilus under-pinene stress. J. For. Res. 2018. [CrossRef]

33. Kong, J.O.; Park, I.K.; Choi, K.S.; Shin, S.C.; Ahn, Y.J. Nematicidal and propagation activities of thyme red and white oil compounds toward Bursaphelenchus xylophilus (Nematoda: Parasitaphelenchidae). J. Nematol. 2007, 39, 237. [PubMed]

34. Croteau, R.; Kutchan, T.M.; Lewis, N.G. Natural products (secondary metabolites). Biochem. Mol. Biol. Plants 2000, 24, 1250-1319.

35. Langenheim, J.H. Higher plant terpenoids: A phytocentric overview of their ecological roles. J. Chem. Ecol. 1994, 20, 1223-1280. [CrossRef] [PubMed]

36. Takeuchi, Y.; Kanzaki, N.; Futai, K. Volatile compounds in pine stands suffering from pine wilt disease: Qualitative and quantitative evaluation. Nematology 2006, 8, 869-879.

37. Yan, X.; Cheng, X.Y.; Wang, Y.S.; Luo, J.; Mao, Z.C.; Ferris, V.R.; Xie, B.Y. Comparative transcriptomics of two pathogenic pinewood nematodes yields insights into parasitic adaptation to life on pine hosts. Gene 2012, 505, 81-90. [CrossRef] [PubMed]

38. Thiel, L.; Hendricks, F. Study into the Establishment of an Aroma and Fragrance Fine Chemicals Value Chain in South Africa, Part III: Aroma Chemicals Derived from Petrochemical Feedstocks, Tender Number T79/07/03; Government Tender Bulletins; National Economic Development and Labor Council: Pretoria, South Africa, 2004.

39. Espada, M.; Silva, A.C.; van den Akker, S.E.; Cock, P.J.; Mota, M.; Jones, J.T. Identification and characterization of parasitism genes from the pinewood nematode Bursaphelenchus xylophilus reveals a multilayered detoxification strategy. Mol. Plant Pathol. 2016, 17, 286-295. [CrossRef] [PubMed]

40. Cheng, X.Y.; Tian, X.L.; Wang, Y.S.; Lin, R.M.; Mao, Z.C.; Chen, N.; Xie, B.Y. Metagenomic analysis of the pinewood nematode microbiome reveals a symbiotic relationship critical for xenobiotics degradation. Sci. Rep. 2013, 3, 1869. [CrossRef] [PubMed]

41. Shreffler, W.; Magardino, T.; Shekdar, K.; Wolinsky, E. The unc-8 and sup-40 genes regulate ion channel function in Caenorhabditis elegans motorneurons. Genetics 1995, 139, 1261-1272.

42. Stein, L.D.; Bao, Z.; Blasiar, D.; Blumenthal, T.; Brent, M.R.; Chen, N.; Chinwalla, A.; Clarke, L.; Clee, C.; Coghlan, A. The genome sequence of Caenorhabditis briggsae: A platform for comparative genomics. PLoS Biol. 2003, 1, E45. [CrossRef]

43. Consortium, C.E.S. Genome sequence of the nematode C. elegans: A platform for investigating biology. Science 1998, 282, 2012-2018. 
44. Ingham, P.W.; Nakano, Y.; Seger, C. Mechanisms and functions of Hedgehog signalling across the metazoa. Nat. Rev. Genet. 2011, 12, 393. [CrossRef] [PubMed]

45. Nüssleinvolhard, C.; Wieschaus, E. Mutations affecting segment number and polarity in Drosophila. Nature 1980, 287, 795-801. [CrossRef]

46. Mohler, J. Requirements for hedgehog, a segmental polarity gene, in patterning larval and adult cuticle of Drosophila. Genetics 1988, 120, 1061-1072. [PubMed]

47. Kang, J.S.; Lee, H.; Moon, I.S.; Lee, Y.; Koh, Y.H.; Je, Y.H.; Lee, S.H. Construction and characterization of subtractive stage-specific expressed sequence tag (EST) libraries of the pinewood nematode Bursaphelenchus xylophilus. Genomics 2009, 94, 70-77. [CrossRef] [PubMed]

48. Choi, I.; Kim, J.; Shin, S.; Park, I. Nematicidal activity of monoterpenoids against the pine wood nematode (Bursaphelenchus xylophilus). Russ. J. Nematol. 2007, 15, 35.

49. Wang, L.; Feng, Z.; Wang, X.; Wang, X.; Zhang, X. DEGseq: An R package for identifying differentially expressed genes from RNA-seq data. Bioinformatics 2010, 26, 136-138. [CrossRef] [PubMed]

50. Storey, J.D.; Tibshirani, R. Statistical significance for genomewide studies. Proc. Natl. Acad. Sci. USA 2003, 100, 9440-9445. [CrossRef] [PubMed]

51. Conesa, A.; Götz, S.; García-Gómez, J.M.; Terol, J.; Talón, M.; Robles, M. Blast2GO: A universal tool for annotation, visualization and analysis in functional genomics research. Bioinformatics 2005, 21, 3674-3676. [CrossRef] [PubMed]

52. Conesa, A.; Götz, S. Blast2GO: A comprehensive suite for functional analysis in plant genomics. Int. J. Plant Genom. 2008, 2008, 619832. [CrossRef]

53. Ye, J.; Fang, L.; Zheng, H.; Zhang, Y.; Chen, J.; Zhang, Z.; Wang, J.; Li, S.; Li, R.; Bolund, L. WEGO: A web tool for plotting GO annotations. Nucleic Acids Res. 2006, 34, W293. [CrossRef]

54. Su, Y.L.; Li, J.M.; Li, M.; Luan, J.B.; Ye, X.D.; Wang, X.W.; Liu, S.S. Transcriptomic analysis of the salivary glands of an invasive whitefly. PLoS ONE 2012, 7, e39303. [CrossRef] [PubMed]

55. Yin, Y.; Martin, J.; Abubucker, S.; Scott, A.L.; Mccarter, J.P.; Wilson, R.K.; Jasmer, D.P.; Mitreva, M. Intestinal transcriptomes of nematodes: Comparison of the parasites Ascaris suum and Haemonchus contortus with the free-living Caenorhabditis elegans. PLoS Neglect. Trop. Dis. 2008, 2, e269. [CrossRef] [PubMed]

56. Li, X.; Sun, H.; Pei, J.; Dong, Y.; Wang, F.; Chen, H.; Sun, Y.; Wang, N.; Li, H.; Li, Y. De novo sequencing and comparative analysis of the blueberry transcriptome to discover putative genes related to antioxidants. Gene 2012, 511, 54-61. [CrossRef] [PubMed]

57. Livak, J.K.; Schmittgen, T.D. Analysis of relative gene expression data using real-time quantitative PCR and the 2(-Delta Delta C(T)) Method. Methods 2001, 25, 402-408. [CrossRef] [PubMed] 